\title{
Distributed optimization decomposition for joint economic dispatch and frequency regulation
}

\author{
Desmond Cai*, Enrique Mallada $^{\dagger}$ and Adam Wierman ${ }^{\dagger}$
}

\begin{abstract}
Economic dispatch and frequency regulation are typically viewed as fundamentally different problems in power systems, and hence are typically studied separately. In this paper, we frame and study a joint problem that optimizes both slow timescale economic dispatch resources and fast timescale frequency regulation resources. We provide sufficient conditions under which the joint problem can be decomposed without loss of optimality into slow and fast timescale problems. These slow and fast timescale problems have appealing interpretations as the economic dispatch and frequency regulation problems respectively. Moreover, the fast timescale problem can be solved using a distributed algorithm that preserves the stability of the network during transients. We also apply this optimal decomposition to propose an efficient market mechanism for economic dispatch that coordinates with frequency regulation.
\end{abstract}

\section{INTRODUCTION}

One of the main objectives of every power system operator is to schedule power generation to meet demand at every time instant [1]-[3]. This is a challenging task that seeks to schedule generators in a cost-efficient manner while also respecting their limitations (e.g., ramp constraints and capacity constraints) and responding rapidly to any supplydemand imbalances that may emerge (e.g., generator outages and line outages). To make matters more complex, slow timescale control is typically performed using market mechanisms while fast timescale control is done via engineered controllers.

The complexity of this global system operation problem means that it is typically broken up into to separate subproblems - (slow timescale) economic dispatch and (fast timescale) frequency regulation - which are studied independently of each other.

Economic dispatch operates at a slow timescale (intervals of 5 minutes or longer) and focuses on efficiency with respect to costs. In particular, the economic dispatch problem seeks to optimally schedule generators so that the total generation cost is minimized subject to line limits and generation capacity and ramping constraints. Economic dispatch has a long history [1], [4]-[8]. It is currently implemented using a market mechanism known as supply function bidding. In this mechanism, generators submit supply functions to the system operator which specify (as a function of price) the amount a generator is willing to produce. The system operator uses those bids to construct the implied cost functions and solves a centralized optimization problem (over single or multiple time periods) to schedule generators in a way that

\footnotetext{
*Desmond Cai is with Department of Electrical Engineering, California Institute of Technology. Email: wccai@caltech.edu

${ }^{\dagger}$ Enrique Mallada and Adam Wierman are with the Department of Computing and Mathematical Sciences, California Institute of Technology. Email: \{mallada, adamw\}@caltech. edu. This work was supported in part by NSF grants CNS-1319820, EPAS-1307794.

The authors would like to thank Steven H. Low from Caltech for insightful discussions.
}

minimizes system costs while meeting demand and slow timescale operating constraints (including line limits, generation capacity constraints, generation ramping constraints, minimum generation constraints, security constraints, etc.). A centralized market implementation is important both to collect bid costs from generators and also because physical laws of power flows impose coupled constraints between generators.

Frequency regulation operates at a fast timescale (from 30 seconds to a few minutes) and focuses on stability rather than efficiency. In particular, the operator seeks to compensate the remaining imbalance between generation and demand, which drives a deviation from the nominal frequency, by quickly rescheduling fast ramping generators. Frequency regulation has a long history [2], [9], [10]. It is currently implemented by a mechanism known as Automatic Generation Control (AGC), which aims to rebalance power and restore the nominal frequency within independent control areas using local measurements. Within each area, AGC uses information on frequency deviation and inter-area flows to compute the necessary change in power needed to rebalance supply and demand in the system, and allocates this change among different generators based on the market clearing allocations from the last economic dispatch execution [1].

\section{A. Contributions of this paper}

While economic dispatch and frequency regulation each, individually, have large and active literatures; these literatures are almost completely disparate. To this point, there has been no rigorous analysis of whether the combination of economic dispatch and frequency regulation solves the global system operator's goal of managing generation resources in order to minimize cost while maintaining stability.

The goal of this paper is to initiate such a study. In particular, we seek to understand when the combination of economic dispatch and frequency regulation optimally solves the global system operator's problem. More generally, we seek to understand when the global system operator's problem can be decomposed, without loss of optimality, into subproblems corresponding to economic dispatch and frequency regulation.

Our main result provides an initial answer. In the context of a DC power flow model and two classes of generators (peakers and baseloads), we show that the global system operator's problem can be decomposed into two subproblems corresponding to economic dispatch and frequency regulation without loss of optimality as long as the generation schedule induces no congestion on average and the time-average prices within each slow timescale interval (used by economic dispatch) are zero (Theorem 1).

This theorem can be viewed as providing a first-principles justification for the current separation of the economic dispatch and frequency regulation problems. Furthermore, 
it provides a guide for the design of market mechanisms for economic dispatch and control policies for frequency regulation since it highlights a sufficient condition for such algorithms to jointly solve the global problem.

In the case of frequency regulation, the form of the problem that emerges from the optimization decomposition differs in important ways from existing frequency regulation operations (Section IV). First, the frequency regulation controller proposed in this paper (which builds on [11]), uses information on generators' costs to drive the power system to an operating point that minimizes its costs. On the other hand, existing approaches use participation factors from the latest economic dispatch run to drive the power system to a stable operating point. Since participation factors might not reflect actual generation costs, the resulting allocation might not be optimal (from the perspective of cost minimization).

In the case of economic dispatch, we illustrate that the form of the problem that emerges from the optimization decomposition can be solved using a market implementation based on supply function bidding (Section V), similar to existing operations. However, our proposed mechanism differs from existing operations in that we allocate frequency regulation resources optimally and we do so without requiring additional communication in the market. Existing operations use economic dispatch LMPs to directly compensate fast timescale frequency regulation resources, where the latter are allocated using engineered controllers without regards to generation costs. The decomposition in this paper suggests that, instead, supply functions submitted at the economic dispatch timescale should be used to allocate frequency regulation resources via the distributed algorithm in Section IV. Our main result in Section V shows that, if the conditions required for optimal decomposition hold, then the competitive equilibrium of our proposed mechanism leads to efficient (optimal) operation.

\section{SySTEM MODEL}

Our aim in this paper is to understand when the system operator's global objective can be decomposed into subproblems that correspond to economic dispatch and frequency regulation. To this end, we formulate a model of the global objective that includes the joint objectives of economic dispatch and frequency regulation. In particular, our model considers the balancing of supply and demand at both the economic dispatch and frequency regulation timescales. We focus on a DC power flow model and consider two generation types (peakers and baseloads) which differ in the responsiveness they can provide.

\section{A. Network model}

Throughout, we consider a finite time horizon partitioned into $K$ discrete intervals indexed by $k \in \mathcal{K}$ where $\mathcal{K}=$ $\{0,1, \ldots, K-1\}$. In principle, the length of each time period $k$ may range from as little as seconds to as long as minutes. However, in this paper, we focus on the case where the length of each time period is on the order tens of seconds.

We consider a connected network with $N$ nodes and $L$ links. Let $\mathcal{N}=\{1,2, \ldots, N\}$ and $\mathcal{L}=\{1,2, \ldots, L\}$ denote the set of nodes and links respectively. We index nodes by $n \in \mathcal{N}$ and links by $l \in \mathcal{L}$. Without loss of generality, we assign each link $l \in \mathcal{L}$ an arbitrary orientation and we let $i(l) \in \mathcal{N}$ and $j(l) \in \mathcal{N}$ denote the tail and head of the link respectively.
We assume that each node $n$ has a deterministic inelastic demand $d_{k, n}>0$ in period $k$. We assume that demand $d_{k, n}$ at each node is never constant over adjacent time periods. We also assume that each node $n$ has two generators which we refer to as peaker and baseload; and we denote denote their production quantities in period $k$ by $q_{k, n}^{p}$ and $q_{k, n}^{b}$ respectively. Let the vectors $d_{k}:=\left(d_{k, 1}, \ldots, d_{k, N}\right)$, $q_{k}^{p}:=\left(q_{k, 1}^{p}, \ldots, q_{k, N}^{p}\right)$, and $q_{k}^{b}:=\left(q_{k, 1}^{b}, \ldots, q_{k, N}^{p}\right)$. Then the vector of injections at all nodes in period $k$ is given by $q_{k}^{p}+q_{k}^{b}-d_{k}$.

The supply-demand balance constraints are given by:

$$
\mathbf{1}^{\top}\left(q_{k}^{b}+q_{k}^{p}-d_{k}\right)=0, \quad k \in \mathcal{K},
$$

where $\mathbf{1} \in \mathbb{R}^{N}$ denotes the vector of all ones.

We adopt the DC power flow model for line flows. Let $\theta_{k, n}$ to denote the phase angle of node $n$ in period $k$. For a link $l \in \mathcal{L}$, let $p_{k, l}$ denote the power flow on the line in period $k$, and let $B_{l}$ denote the sensitivity of the flow with respect to changes in the phase difference $\theta_{k, i(l)}-\theta_{k, j(l)}$ in period $k$. Let the vectors $\theta_{k}:=\left(\theta_{k, 1}, \ldots, \theta_{k, N}\right)$ and $p_{k}:=$ $\left(p_{k, 1}, \ldots, p_{k, L}\right)$ and the matrix $B:=\operatorname{diag}\left(B_{1}, \ldots, B_{L}\right)$. Hence, the line flows in period $k$ are given by:

$$
p_{k}=B C^{\top} \theta_{k}
$$

where $C \in \mathbb{R}^{N \times L}$ is the incidence matrix of the directed graph. Note that the injections are given by:

$$
q_{k}^{b}+q_{k}^{p}-d_{k}=C p_{k}=L \theta_{k},
$$

where $L:=C B C^{\top}$.

Note that (1) and (2) are equivalent. This is because, for any set of power injections that satisfy (1), we can always find a $\theta_{k}$ that satisfy (2). Conversely, since $\mathbf{1}^{\top} C=0$, any injections that satisfy (2) also satisfy (1).

Hence, the line flows can be written in terms of the power injections:

$$
p_{k}=B C^{\top} L^{\dagger}\left(q_{k}^{b}+q_{k}^{p}-d_{k}\right),
$$

where $L^{\dagger}$ denotes the pseudo-inverse of $L$. Let $H:=$ $B C^{\top} L^{\dagger}$. Let $f_{l}$ denote the capacity of line $l$ and let the vector $f:=\left(f_{1}, \ldots, f_{L}\right)$. Then the line flow constraints are given by:

$$
-f \leq H\left(q_{k}^{b}+q_{k}^{p}-d_{k}\right) \leq f, \quad k \in \mathcal{K} .
$$

\section{B. Generation types}

As mentioned above, we consider two types of generation - peakers and baseloads - where peaker refers to more responsive generation and baseload refers to less responsive generation. Recall that $q_{n, k}^{p}$ and $q_{n, k}^{b}$ denote the production quantities of the peaker and baseload respectively at node $n$ in period $k$.

We assume that the peaker and baseload at node $n$ incur costs of $c_{n}^{p}\left(q_{k, n}^{p}\right)$ and $c_{n}^{b}\left(q_{k, n}^{b}\right)$ respectively for their productions in period $k$ where $c_{n}^{p}: \mathbb{R}_{+} \rightarrow \mathbb{R}_{+}$and $c_{n}^{b}: \mathbb{R}_{+} \rightarrow \mathbb{R}_{+}$ are continuously differentiable and convex. We also assume that the peaker and baseload have capacities $\bar{q}_{n}^{p} \in \mathbb{R}_{+}$ and $\bar{q}_{n}^{b} \in \mathbb{R}_{+}$respectively and that $c_{n}^{p}\left(q_{k, n}^{p}\right) \rightarrow+\infty$ and $c_{n}^{b}\left(q_{k, n}^{b}\right) \rightarrow+\infty$ as $q_{k, n}^{p} \rightarrow\left\{0, \bar{q}_{n}^{p}\right\}$ and $q_{k, n}^{b} \rightarrow\left\{0, \bar{q}_{n}^{b}\right\}$ respectively. Let the vectors $\bar{q}^{p}:=\left(\bar{q}_{1}^{p}, \ldots, \bar{q}_{N}^{p}\right)$ and $\bar{q}^{b}:=$ $\left(\bar{q}_{1}^{b}, \ldots, \bar{q}_{N}^{b}\right)$. These capacity constraints are given by:

$$
\begin{array}{ll}
0 \leq q_{k}^{p} \leq \bar{q}^{p}, & k \in \mathcal{K} ; \\
0 \leq q_{k}^{b} \leq \bar{q}^{b}, & k \in \mathcal{K} .
\end{array}
$$


We model the fact that baseloads are less responsive than peakers using two features.

First, we assume that peakers may change production levels every time period while baseloads change production levels every $S$ time periods where $K \bmod S=0$. Let $\mathcal{S}$ denote the set of time periods in which baseloads may change production levels, i.e. $\mathcal{S}=\{0, S, 2 S, \ldots, K-S\}$. For each $k \in \mathcal{S}$, let $\mathcal{K}_{k}=\{k, k+1, \ldots, k+S-1\}$ denote the set of time periods in the corresponding length-S interval during which baseload productions are constant. For each $k \in \mathcal{S}$ and $k^{\prime} \in \mathcal{K}_{k}$, define $s\left(k^{\prime}\right):=S\lfloor(k-1) / S\rfloor=k$. Then the constraints on baseloads' decisions are represented by:

$$
q_{k}^{b}=q_{s(k)}^{b}, \quad k \in \mathcal{K}
$$

\section{System operator's objective}

The global system operator's objective is to dispatch the baseload and peaker generation in order to minimize the total cost needed to satisfy demand and operating constraints. This is formalized as follows.

$$
\begin{aligned}
& \text { SYSTEM : minimize } \sum_{k} \sum_{n}\left(c_{n}^{b}\left(q_{n, k}^{b}\right)+c_{n}^{p}\left(q_{n, k}^{p}\right)\right) \\
& \text { over } q_{k}^{b}, q_{k}^{p}, \quad k \in \mathcal{K} \text {; } \\
& \text { s.t. (1), (3), (4) - (6). }
\end{aligned}
$$

We assume throughout that this optimization is feasible.

In addition to the constraints highlighted above there are many practical issues that must be taken into account in any feasible solution. At the fast time scale, system demand must be measured precisely and controls implemented such that generators do not lose synchrony. Hence, a practical implementation must include mechanisms to restore frequency and preserve grid stability. A practical implementation must also include market mechanisms to extract cost functions from generators.

The current practice in economic dispatch is to clear the market without fast time scale supply-demand constraints. Instead, these fast time scale constraints are implemented using frequency regulation controls without consideration of the costs of generation. In Section III, we provide an architectural decomposition of the global objective into slow time scale and fast time scale subproblems without loss of optimality under certain conditions. We propose a distributed frequency regulation algorithm to implement the solution to the fast time scale subproblem and a market mechanism to extract cost functions for the slow time scale subproblem. We address these in Sections IV and V respectively.

\section{Lagrangian relaxation}

Crucial to our main result is the relaxation of the supplydemand balance constraints and line flow constraints of the system operator's objective. In particular, let $\lambda_{k}$ denote the Lagrange multipliers associated with the supply-demand balance constraint (1) in period $k$ and let $\mu_{k, l}^{-}, \mu_{k, l}^{+}$denote the Lagrange multipliers associated with the negative and positive line flow constraints (3) respectively in period $k$. Let $\mu_{k}^{-}:=\left(\mu_{k, 1}, \ldots, \mu_{k, L}\right)$ and $\mu_{k}^{+}:=\left(\mu_{k, 1}, \ldots, \mu_{k, L}\right)$. Let $x:=\left(q_{k}^{b}, q_{k}^{p}\right)_{k \in \mathcal{K}}$ and $y:=\left(\lambda_{k}, \mu_{k}^{-}, \mu_{k}^{+}\right)_{k \in \mathcal{K}}$ Then, the
Lagrangian associated with relaxing those constraints is:

$$
\begin{aligned}
L(x, y)= & \sum_{k \in \mathcal{K} \backslash \mathcal{S}} f\left(q_{k}^{p},\left(\lambda_{k}, \mu_{k}^{-}, \mu_{k}^{+}\right)\right) \\
& +\sum_{k \in \mathcal{S}} g\left(\left(q_{k}^{b}, q_{k}^{p}\right),\left(\lambda_{k^{\prime}}, \mu_{k^{\prime}}^{-}, \mu_{k^{\prime}}^{+}\right)_{k^{\prime} \in \mathcal{K}_{k}}\right),
\end{aligned}
$$

where:

$$
\begin{aligned}
& f\left(q_{k}^{p},\left(\lambda_{k}, \mu_{k}^{-}, \mu_{k}^{+}\right)\right) \\
& :=\sum_{n} c_{n}^{p}\left(q_{n, k}^{p}\right)-\lambda_{k} \mathbf{1}^{\top} q_{k}^{p}-\left(\mu_{k}^{-}-\mu_{k}^{+}\right)^{\top} H q_{k}^{p}, \\
& g\left(\left(q_{k}^{b}, q_{k}^{p}\right),\left(\lambda_{k^{\prime}}, \mu_{k^{\prime}}^{-}, \mu_{k^{\prime}}^{+}\right)_{k^{\prime} \in \mathcal{K}_{k}}\right) \\
& :=\sum_{n}\left(S c_{n}^{b}\left(q_{n, k}^{b}\right)+c_{n}^{p}\left(q_{n, k}^{p}\right)\right)-\lambda_{k} \mathbf{1}^{\top} q_{k}^{p} \\
& \quad-\sum_{k^{\prime} \in \mathcal{K}_{k}} \lambda_{k^{\prime}} \mathbf{1}^{\top} q_{k}^{b}-\sum_{k^{\prime} \in \mathcal{K}_{k}}\left(\mu_{k^{\prime}}^{-}-\mu_{k^{\prime}}^{+}\right)^{\top} H q_{k}^{b} .
\end{aligned}
$$

Since $S Y S T E M$ is convex and the constraints are linear, it is equivalent to the following problem:

$$
\max _{y: \mu_{k}^{-} \geq 0, \mu_{k}^{+} \geq 0, k \in \mathcal{K}} \min _{x:(4)-(6)} L(x, y) .
$$

We refer to the optimal $y$ in this problem as the optimal Lagrange multipliers.

\section{ARChitectural DeCOMposition}

Our goal in this paper is to understand, from first principles, how the structure of the global system operator's problem can guide the architecture of control for power systems. To that end, our main result is a decomposition of the global system operator's problem that exposes fast timescale and slow timescale control problems and that serves as guides for the design of market mechanisms for economic dispatch and control policies frequency regulation. Importantly, the decomposition we derive identifies a rigorous connection between economic dispatch and frequency regulation that ensures, under certain conditions, that the combination solves the global system operator's problem.

Theorem 1. Let $\left(q_{k}^{b *}, q_{k}^{p *}\right)_{k \in \mathcal{K}}$ denote the optimal baseload and peaker productions of SYSTEM and let $\left(\lambda_{k}^{*}, \mu_{k}^{-*}, \mu_{k}^{+*}\right)_{k \in \mathcal{K}}$ denote the optimal Lagrange multipliers. Suppose that, for each $k \in \mathcal{S}$, we have:

(a) $q_{k}^{b *}=0$, or;

(b) $\sum_{k^{\prime} \in \mathcal{K}_{k} \backslash\{k\}} H^{\top}\left(\mu_{k^{\prime}}^{-*}-\mu_{k^{\prime}}^{+*}\right)=0$ and $q_{k}^{p *}=0$, or;

(c) $\sum_{k^{\prime} \in \mathcal{K}_{k} \backslash\{k\}} H^{\top}\left(\mu_{k^{\prime}}^{-*}-\mu_{k^{\prime}}^{+*}\right)=0$ and
$\sum_{k^{\prime} \in \mathcal{K}_{k} \backslash\{k\}} \lambda_{k^{\prime}}^{*}=0$.

Then $\left(q_{k}^{b *}, q_{k}^{p *}\right)_{k \in \mathcal{S}}$ is the optimal solution to the following problem:

$$
\begin{aligned}
& E D: \quad \min \quad \sum_{k \in \mathcal{S}} \sum_{n}\left(S c_{n}^{b}\left(q_{k, n}^{b}\right)+c_{n}^{p}\left(q_{k, n}^{p}\right)\right) \\
& \text { over } \quad q_{k}^{b}, q_{k}^{p}, \quad k \in \mathcal{S} ; \\
& \text { s.t. } \quad \mathbf{1}^{\top}\left(q_{k}^{b}+q_{k}^{p}-d_{k}\right)=0, \quad k \in \mathcal{S} ; \\
&-f \leq H\left(q_{k}^{b}+q_{k}^{p}-d_{k}\right) \leq f, \quad k \in \mathcal{S} ; \\
& 0 \leq q_{k}^{b} \leq \bar{q}^{b}, \quad k \in \mathcal{S} ; \\
& 0 \leq q_{k}^{p} \leq \bar{q}^{p}, \quad k \in \mathcal{S} .
\end{aligned}
$$


And, for each $k \in \mathcal{K} \backslash \mathcal{S}, q_{k}^{p *}$ is the optimal solution to the following problem:

$$
\begin{array}{rll}
F R_{k}: \quad \min & \sum_{n} c_{n}^{p}\left(q_{k, n}^{p}\right) \\
\text { over } \quad & q_{k}^{p} ; \\
\text { s.t. } \quad & \mathbf{1}^{\top}\left(q_{s(k)}^{b *}+q_{k}^{p}-d_{k}\right)=0 ; \\
& -f \leq H\left(q_{s(k)}^{b *}+q_{k}^{p}-d_{k}\right) \leq f ; \\
& 0 \leq q_{k}^{p} \leq \bar{q}^{p} ; \\
\text { given } \quad & q_{s(k)}^{b *} .
\end{array}
$$

The proof of Theorem 1 is given in the Appendix. At a high level, the result follows from a dual decomposition of the system operator's problem into separate problems that can be interpreted as problems on two different time scales. Note that the subproblems $E D$ and $F R_{k}$ can be solved in a modular fashion with causal communication. In particular, $E D$ will be solved first once and then only the optimal baseload generations are needed as setpoints to solve $F R_{k}$.

We denote the two sub-optimizations in Theorem 1 by $E D$ and $F R_{k}$ because they can be interpreted as the economic dispatch and frequency regulation components, respectively, of existing operations. The correspondence is immediate in the case of $E D$ and we discuss how the decomposition leads to improved market mechanisms for economic dispatch based on supply function bidding in Section V. However, the correspondence may not be as clear in the case of $F R_{k}$. We show in Section IV that $F R_{k}$ can in fact be solved via distributed frequency regulation algorithms, although these algorithms deviate from current practice since current approaches typically do not optimize for generation costs.

Perhaps the most important part of Theorem 1 to discuss is the conditions required for the proposed decomposition to be optimal. The interpretation of these conditions is as follows. Condition (a), that $q_{k}^{b *}=0$, corresponds to settings where baseloads are always more expensive than peakers; thus implying that it is never optimal to use baseload generation (since they are also less responsive). Similarly, the condition that $q_{k}^{p *}=0$ corresponds to settings where peakers are more expensive than baseloads, thus implying that it is never optimal to use peaker generation when there is an option to use baseload generation. The conditions on $\mu_{k}^{-*}$ and $\mu_{k}^{+*}$ can be interpreted as guaranteeing that the generation schedule in period $k \in \mathcal{S}$ induces no congestion (on average) on the aggregate network over each slow timescale interval. Finally, the condition on $\lambda_{k}^{*}$ can be interpreted as guaranteeing that the average energy prices over each slow timescale interval are zero, thus implying that generation at the slow timescale can be optimally scheduled by solving a myopic problem.

Given the above, the most interesting cases seem to be (b) and (c), which highlight that time-averaged prices are the primary determinant of whether the global system operator's problem can be decomposed into separate timescales without loss of optimality. We might expect the average energy prices over each slow timescale interval to be zero or very small if the slow timescale demand $d_{k}$ for $k \in \mathcal{S}$ is a zero-mean forecast of actual demands over the slow timescale interval. In practice, congestion typically occurs over a very small minority of lines, and hence we can expect the average aggregation congestion prices for most nodal injections to be zero or very small relative to the costs of energy.
Note that Theorem 1 is close in spirit to work in communication networks that uses optimization decomposition to justify and optimize protocol layering, e.g., see [12][15]. This work provides a rigorous way to think about the architectural design of communication networks. Though similar in spirit, Theorem 1 highlights a crucial difference between communication networks and power networks. In particular, in communication networks, different layers in the protocol stack may coordinate by communicating primal and dual variables when solving the sub-optimizations. However, such mechanics do not apply to power networks since the nature of the timescales implies that sub-optimizations cannot have non-causal dependencies.

Finally, note that Theorem 1 focuses only on the question of when the system operator's problem can be decomposed without loss of optimality. An important extension of this work is to understand the efficiency loss in cases where this decomposition is no longer optimal.

\section{Distributed Frequency Regulation}

The goal of this section is to illustrate that the solution of $F R_{k}$ can be implemented using distributed frequency regulation controllers that respect the engineering constraints of the system. Besides achieving optimality, a practical solution should introduce changes on the power scheduling that preserve the network stability; it should be robust to unexpected system events; and it should be able to quickly aggregate distributed network information in order to guarantee constraints (7a) and (7b).

In this section we provide a distributed algorithm that not only solves $F R_{k}$, but also cleverly uses network dynamics in order to aggregate the necessary information. The algorithm can be interpreted as performing distributed frequency regulation by sending different regulation signals to each bus. Importantly, the algorithm only requires local information and can be shown to preserve the stability of the network.

\section{A. A dynamic model}

Before introducing our algorithm for distributed frequency regulation, we first need to add dynamics to our system model. In particular, we describe a model for system changes within a single time period $k$ in the following.

Let $t$ denote the time evolution within the time period and assume without loss of generality that $t \in(k, k+1]$. Let $q_{k}^{p}(t):=\left(q_{k, 1}^{p}(t), \ldots, q_{k, N}^{p}(t)\right)$ denote the quantities generated by the peakers at time $t$. We assume that baseloads and demand do not change within the time period. Hence, baseloads generate $q_{k}^{b}$ and demand consumes $d_{k}$. Then, the system changes within the time period are governed by the swing equations which we assume to have the following form:

$$
\begin{aligned}
M \dot{\omega}_{k}(t) & =q_{k}^{b}+q_{k}^{p}(t)-d_{k}-D \omega_{k}(t)-L \theta_{k}(t) ; \\
\dot{\theta}_{k}(t) & =\omega_{k}(t),
\end{aligned}
$$

where $\omega_{k}(t):=\left(\omega_{k, 1}(t), \ldots, \omega_{k, N}(t)\right)$ are the frequency deviations from the nominal value at time $t, \theta_{k}(t):=$ $\left(\theta_{k, 1}(t), \ldots, \theta_{k, N}(t)\right)$ are the phase angles at time $t, M:=$ $\operatorname{diag}\left(M_{1}, \ldots, M_{N}\right)$ where $M_{n}$ is the aggregate inertia of baseload and peaker $n$, and $D:=\operatorname{diag}\left(D_{1}, \ldots, D_{N}\right)$ where $D_{n}$ is the aggregate damping of baseload and peaker $n$. Here, the notation $\dot{x}$ denotes the time derivative, i.e. $\dot{x}=d x / d t$.

Equation (8) is a linearized version of the nonlinear network dynamics widely adopted by the power systems 
community, e.g., [2], [16]. We refer the reader to [17] for a detailed explanation of its derivation.

\section{B. Current practice}

In today's grid, frequency regulation is implemented using a control scheme known as Automatic Generation Control (AGC) that is executed between two different executions of $E D$. To implement AGC, the power grid is divided into several control areas, each one of them in charge of restoring the frequency to its nominal value and compensating its own supply demand imbalance.

This is achieved for a given area $A$ by generating a unique control signal known as Area Control Error $\left(A C E_{A}\right)$ given by

$$
A C E_{A}(t)=K_{A} \omega_{A}(t)+\Delta \operatorname{Tie}_{A}(t),
$$

where $\omega_{A}(t)$ represents the average frequency in area $A$, $K_{A}$ is the frequency bias setting and $\Delta \operatorname{Tie}_{A}(t)$ represents the net area interchange deviation with respect to the interchange scheduled by $E D$. The signal $A C E_{A}(t)$ is then sent through a proportional-integral controller that outputs the total amount of power generation that needs to be corrected.

Finally, the total change in power needed is distributed among generators using participating factors that are proportional to the nodal prices $\lambda_{s(k), n}+e_{n}^{\top} H^{\top}\left(\mu_{s(k)}^{-}-\mu_{s(k)}^{+}\right)$ in $E D$ where $e_{n}$ denotes a unit vector with a 1 in the $n$th component.

There are a number of sources of inefficiency in this approach. First, AGC relies on information from the economic dispatch problem that is likely out of date due to the timescale difference. Second, AGC does not satisfy the thermal limits at fast timescales. Third, AGC requires the definition of self-balancing areas which are forced to independently rebalance supply and demand within each area [1].

These problems have recently been acknowledged by the research community [18], [19]. The main solution strategy proposed is the use distributed algorithms that dynamically adapt to power fluctuations in order to rebalance the system while minimizing the total generation cost. While these solutions can successfully adapt to rapid changes on the network, they do not respect the ramping constraints of baseline generator and cannot be implemented together with economic dispatch.

\section{Distributed frequency regulation}

In contrast to AGC, we now introduce a distributed, continuous-time algorithm that provably solves $F R_{k}$, and thus (by Theorem 1) integrates with economic dispatch to optimally solve the system operator's problem while satisfying the thermal line limits.

Our solution is based on a novel reverse and forward engineering approach for distributed control design in power systems [11], [18], [20], [21]. The key step in this approach is to formulate an optimization problem whose primal-dual algorithm includes the power network dynamics as part of it and where the remaining part can be implemented using distributed communication and computation [11].

The algorithm operates as follows. Each peaker $n$ updates its power generation using

$$
q_{k, n}^{p}(t)=\left[c_{n}^{p^{\prime}-1}\left(\omega_{k, n}(t)+\lambda_{k, n}(t)\right)\right]_{0}^{\bar{q}_{n}^{p}},
$$

where $c_{n}^{p^{\prime}}(x)=\frac{\partial}{\partial x} c_{n}^{p}(x)$ and $c_{n}^{p^{\prime}-1}$ denotes its inverse. The projection $\left[u_{i}\right]_{0}^{\bar{q}_{n}^{p}}$ ensures that $u_{i}$ is always within $\left[0, \bar{q}_{n}^{p}\right]$, and $\lambda_{k, n}(t)$ is a control signal generated using:

$$
\begin{aligned}
D F R: \dot{\lambda}_{k}(t) & =\zeta^{\lambda}(t)\left(q_{k}^{b *}-d_{k}+q_{k}^{p}(t)-L \phi_{k}(t)\right) ; \\
\dot{\mu}_{k}^{+}(t) & =\zeta^{\mu^{+}}\left[B C^{\top} \phi_{k}(t)-f\right]_{\mu_{k}^{+}}^{+} \\
\dot{\mu}_{k}^{-}(t) & =\zeta^{\mu^{-}}\left[-f-B C^{\top} \phi_{k}(t)\right]_{\mu_{k}^{-}}^{+} \\
\dot{\phi}_{k}(t) & =\chi^{\phi}\left(L \lambda_{k}(t)-C B\left(\mu_{k}^{+}(t)-\mu_{k}^{-}(t)\right)\right)
\end{aligned}
$$

where $\zeta^{\lambda}:=\operatorname{diag}\left(\zeta_{1}^{\lambda}, \ldots, \zeta_{N}^{\lambda}\right), \zeta^{\mu^{+}}:=\operatorname{diag}\left(\zeta_{1}^{\mu^{+}}, \ldots, \zeta_{L}^{\mu^{+}}\right)$, $\zeta^{\mu^{-}}:=\operatorname{diag}\left(\zeta_{1}^{\mu^{-}}, \ldots, \zeta_{L}^{\mu^{-}}\right)$and $\chi^{\phi}:=\operatorname{diag}\left(\chi_{1}^{\phi}, \ldots, \chi_{N}^{\phi}\right)$ denote the respective control gains. Given vectors $x, y \in$ $\mathbb{R}^{M}$ and $\mathcal{M}=\{1, \ldots, M\}$, the element-wise projection $[y]_{x}^{+}$ensures that the dynamics $\dot{x}=[y]_{x}^{+}$have a solution $x(t)$ that remains in the positive orthant. That is, $[y]_{x}^{+}:=$ $\left(\left[y_{m}\right]_{x_{m}}^{+}\right)_{m \in \mathcal{M}}$, with $\left[y_{m}\right]_{x_{m}}^{+}=0$ if $x_{m}=0$ and $y_{m}<0$; $\left[y_{m}\right]_{x_{m}}^{+}=y_{m}$, otherwise.

The proposed solution (9) - (10) can be interpreted as a frequency regulation algorithm in which each peaker receives a different regulation signal (9) depending on its location in the network.

\section{Optimality and convergence}

We now show how the distributed algorithm described above converges to the optimal solution of $F R_{k}$.

To this end, we first modify $F R_{k}$ and define a related problem $F R_{k}^{\prime}$ that can be shown to be equivalent to $F R_{k}$ while, at the same time, making the role of frequency in maintaining supply-demand balance explicit. This is a nontrivial modification of $F R_{k}$ and is crucial to guaranteeing the stability of our distributed algorithm.

$$
\begin{aligned}
F R_{k}^{\prime}: \quad \min & \sum_{n}\left(c_{n}^{p}\left(q_{k, n}^{p}\right)+D_{n} \frac{\omega_{n}^{2}}{2}\right) \\
\text { over } \quad & q_{k}^{p}, \omega_{k}, \theta_{k}, \phi_{k} ; \\
\text { s.t. } \quad & q_{s(k)}^{b *}+q_{k}^{p}-d_{k}-D \omega_{k}=L \theta_{k} ; \\
& q_{s(k)}^{b *}+q_{k}^{p}-d_{k}=L \phi_{k} ; \\
& -f \leq B C^{\top} \phi_{k} \leq f \\
& 0 \leq q^{p} \leq \bar{q}^{p} \\
\text { given } \quad & q_{s(k)}^{b *} .
\end{aligned}
$$

Constraint (11a) makes explicit the fact that, whenever supply and demand do not match, the mismatch is compensated by a change in the frequency. We have also used the equivalent per node supply-demand balance constraint (2) instead of the aggregate supply-demand balance constraint (1). Constraint (11b) ensures that the optimal solution satisfies $\omega_{k}^{*}=0$ so that supply and demand are balanced. Constraint (11c) imposes line flow limits. However, instead of using actual line flows $B C^{\top} \theta_{k}$, we impose these limits on virtual flows $B C^{\top} \phi_{k}$.

The next proposition formally relates $F R_{k}$ and $F R_{k}^{\prime}$ and guarantees the optimality of (9) - (10).

Proposition 1 (Optimality). Let $q_{k}^{p * *}$ and $\left(q_{k}^{p *}, \omega_{k}^{*}, \theta_{k}^{*}, \phi_{k}^{*}\right)$ be optimal solutions of $F R_{k}$ and $F R_{k}^{\prime}$ respectively. Then, the following statements are true:

(i) Frequency restoration: $\omega_{k}^{*}=0$; 
(ii) Generation equivalence: $q_{k}^{p * *}=q_{k}^{p *}$;

(iii) Line flow equivalence:

$$
B C^{\top} \theta_{k}^{*}=H\left(q_{s(k)}^{b *}+q_{k}^{p * *}-d_{k}\right) .
$$

Moreover, a vector $\left(q_{k}^{p *}, \omega_{k}^{*}, \theta_{k}^{*}, \phi_{k}^{*}, \lambda^{*}, \mu^{+*}, \mu^{-*}\right)$ is an equilibrium point of (8) - (10) if and only if it is a primal-dual optimal solution of $F R_{k}^{\prime}$.

What remains is to guarantee the convergence of the distributed frequency regulation algorithm.

Proposition 2 (Convergence). Given the distributed frequency regulation scheme (9) - (10) and the network dynamics (8). Then, provided that $c_{n}^{p}(\cdot)$ is twice continuous differentiable with $\left(c_{n}^{p}\right)^{\prime \prime}(\cdot) \geq \alpha>0$ ( $\alpha$-strictly convex), the coupled dynamics (9) - (10) and (8) converge globally to an optimal solution of $F R_{k}$.

The proof of Proposition 2 follows from [11, Theorem 9]. It is easy to show that by substituting the phase representation of the line flows $B C^{T} \theta_{k}$ with $p_{k}$ in $F R_{k}^{\prime}$ and (8), the whole system (9) - (10) and (8) is a primal-dual algorithm of $F R_{k}^{\prime}$. Therefore, Theorem 9 in [11] guarantees global asymptotic convergence to an equilibrium point which by Proposition 1 is an optimal solution of both $F R_{k}^{\prime}$ and $F R_{k}$. We remark the controllers of [11] have additional states, but the proof in this simpler case is identical.

\section{A Market Mechanism for Economic Dispatch}

We now move our attention to the economic dispatch component of the decomposition provided by Theorem 1 . The goal of this section is to illustrate that the solution of the economic dispatch problem formalized in $E D$ can be implemented using a market mechanism based on supply function bidding. The mechanism we propose aligns with current practice, but differs in an important way that ensures proper coordination with frequency regulation, thus avoiding the inefficiency of approaches adopted today.

\section{A. Current practice}

The economic dispatch problem is solved in practice using complicated market mechanisms, see [5], [22]-[24] for an overview. Briefly, existing markets price slow timescale economic dispatch resources using locational marginal prices (LMPs) which are derived from Lagrange multipliers on the energy balance and congestion constraints. As we described in Section IV-B, the LMPs are used to compensate any frequency regulation resources dispatched within each slow timescale interval.

This implementation is adopted, in part, because of the short timescale of frequency regulation (on the order of seconds), which makes it challenging to implement separate markets for each frequency regulation interval. However, inefficiencies arise because the Lagrange multipliers may vary within each slow timescale interval so prices computed from the economic dispatch problem may not provide the appropriate incentives for frequency regulation. Such inefficiencies are becoming more significant due to the growth of renewables [25]-[27].

\section{B. Market Mechanism}

The approach for economic dispatch suggested by the decomposition in Theorem 1 is similar to current practice, but it also provides insight on how to avoid the inefficiency highlighted above.

In particular, we propose a market mechanism that operates on the timescale of economic dispatch but includes an efficient pricing mechanism for fast timescale frequency regulation. Our proposed mechanism is efficient if the conditions for decomposability of the global problem provided in Theorem 1 hold and does not require any more communication than existing market mechanisms.

Concretely, in our proposal the system operator collects supply function bids from generators at the slow timescale and solves the economic dispatch problem as in the current practice. However, instead of compensating frequency regulation resources using slow timescale locational prices, the system operator uses the bids to allocate the peaking resources efficiently at the fast timescale.

1) Assumptions: In this section, we make the following assumptions. We assume that generators are not capacityconstrained, i.e. $\bar{q}^{b}=\bar{q}^{p}=\infty$. We also assume that there exists a continuously differentiable convex function $c: \mathbb{R}_{+} \rightarrow \mathbb{R}_{+}$such that peaker and baseload cost functions are proportional to $c$, that is:

$$
\begin{aligned}
& c_{n}^{p}(\cdot)=\left(1 / \theta_{n}^{p}\right) c(\cdot) ; \\
& c_{n}^{b}(\cdot)=\left(1 / \theta_{n}^{b}\right) c(\cdot),
\end{aligned}
$$

for some $\theta_{n}^{p}, \theta_{n}^{b}>0$. In practice, generators do have capacity constraints. However, these assumptions are imposed primarily to simplify the exposition. Moreover, many studies of electricity markets are performed using quadratic costs [28][30].

2) Supply functions: Each supply function specifies (as a function of price) the amount the generator is willing to produce. We assume that supply functions are chosen from a parameterized family of supply functions. Formally, we assume that a supply function within the parameterized family is represented by a parameter $\theta \in \mathbb{R}_{+}$. Each supply function indicates that, at a price $p$, the generator is willing to supply the quantity $\theta s(p)$, where $s: \mathbb{R}_{+} \rightarrow \mathbb{R}_{+}$is a differentiable function. We define the surrogate cost function associated with this supply function as:

$$
\hat{c}(q, \theta):=(1 / \theta) \int_{0}^{q} s^{-1}(\bar{q}) d \bar{q} .
$$

This definition is motivated by the practice in existing electricity markets. Numerous studies have explored different functional forms of $s$ and their impact on the efficiency of the market, e.g., [6], [24], [31]-[33]. For this paper, we assume that $s(p)=(1 / \theta) c^{-1}(\theta p)$. It follows that:

$$
\hat{c}(q, \theta)=(1 / \theta) \int_{0}^{q} c^{\prime}(\bar{q}) d \bar{q}=(1 / \theta) c(q) .
$$

3) Mechanism: Each baseload and peaker submits supply functions to the system operator and the system operator uses these supply functions to construct surrogate cost functions and clears the market by solving $E D$ and $F R_{k}$ using the surrogate cost functions.

Formally, each baseload $n$ submits a sequence of supply functions $\left(\hat{\theta}_{k, n}^{b}\right)_{k \in \mathcal{S}}$ to the system operator where $\hat{\theta}_{k, n}^{b}$ is its supply function in period $k \in \mathcal{S}$. Each peaker $n$ submits a sequence of supply functions $\left(\hat{\theta}_{k, n}^{p}\right)_{k \in \mathcal{S}}$ to the system operator where $\hat{\theta}_{s(k), n}^{p}$ is its supply function in period $k \in$ 
$\mathcal{K}$. Note that each peaker $n$ only chooses a single supply function $\hat{\theta}_{s(k), n}^{p}$ for all periods $k \in \mathcal{K}_{k}$. Hence, the bids in the economic dispatch timescale are used as bids in the frequency regulation timescale.

We assume that baseloads and peakers are price-takers. Hence, given a sequence of prices $\left(p_{k, n}\right)_{k \in \mathcal{K}}$, each baseload $n$ chooses bids $\left(\hat{\theta}_{k, n}^{b}\right)_{k \in \mathcal{S}}$ to maximize its profit:

$$
\begin{aligned}
& B_{n}: \quad \operatorname{maximize} \sum_{k \in \mathcal{S}} S\left(p_{k, n} \hat{\theta}_{k, n}^{b} s\left(p_{k, n}\right)\right. \\
&\left.-\left(1 / \theta_{n}^{b}\right) c\left(\hat{\theta}_{k, n}^{b} s\left(p_{k, n}\right)\right)\right) \\
& \text { over } \quad \hat{\theta}_{k, n}^{b}, \quad k \in \mathcal{S} ; \\
& \text { s.t. } \quad 0 \leq \hat{\theta}_{k, n}^{b} s\left(p_{k, n}\right), \quad k \in \mathcal{S} ; \\
& \text { given } \quad p_{k, n}, \quad k \in \mathcal{K},
\end{aligned}
$$

and each peaker $n$ chooses bids $\left(\hat{\theta}_{k, n}^{p}\right)_{k \in \mathcal{S}}$ to maximize its profit:

$$
\begin{aligned}
& P_{n}: \quad \text { maximize } \sum_{k \in \mathcal{K}}\left(p_{k, n} \hat{\theta}_{s(k), n}^{p} s\left(p_{k, n}\right)\right. \\
&\left.\quad-\left(1 / \theta_{n}^{p}\right) c\left(\hat{\theta}_{s(k), n}^{p} s\left(p_{k, n}\right)\right)\right) \\
& \text { over } \quad \hat{\theta}_{k, n}^{p}, \quad k \in \mathcal{S} ; \\
& \text { s.t. } \quad 0 \leq \hat{\theta}_{s(k), n}^{p} s\left(p_{k, n}\right), \quad k \in \mathcal{K} ; \\
& \text { given } \quad p_{k, n}, \quad k \in \mathcal{K} .
\end{aligned}
$$

Let $\hat{\theta}_{k}^{b}:=\left(\hat{\theta}_{k, 1}^{b}, \ldots, \hat{\theta}_{k, N}^{b}\right)$ and $\hat{\theta}_{k}^{p}:=\left(\hat{\theta}_{k, 1}^{p}, \ldots, \hat{\theta}_{k, N}^{p}\right)$ denote the vectors of bids in period $k$. Given the bids $\left(\hat{\theta}_{k}^{b}, \hat{\theta}_{k}^{p}\right)_{k \in \mathcal{S}}$, the system operator solves $E D$ for the dispatch that minimizes the surrogate costs. The nodal prices are given by $p_{k}=\lambda_{k}^{*}+H^{\top}\left(\mu_{k}^{-*}-\mu_{k}^{+*}\right)$ where $\lambda_{k}^{*}, \mu_{k}^{-*}, \mu_{k}^{+*}$ are optimal Lagrange multipliers in $E D$. Then, in each fast time scale period $k \in \mathcal{K} \backslash \mathcal{S}$, it implements the frequency regulation algorithm $D F R$ with the surrogate costs functions to drive the system to the optimal solution of $F R_{k}$. The nodal prices are again given by $p_{k}=\lambda_{k}^{*}+H^{\top}\left(\mu_{k}^{-*}-\mu_{k}^{+*}\right)$ but now $\lambda_{k}^{*}, \mu_{k}^{-*}, \mu_{k}^{+*}$ are optimal Lagrange multipliers in $F R_{k}$.

\section{Efficiency}

Given the mechanism described above, our focus is on understanding the efficiency of equilibrium outcomes. The following formalizes the notion of a competitive equilibrium we consider.

Definition 1. We say that $\left(\hat{\theta}_{k}^{b}, \hat{\theta}_{k}^{p}\right)_{k \in \mathcal{S}}$ is a competitive equilibrium if there exists $\left(p_{k}\right)_{k \in \mathcal{K}}$ such that:

(a) For all $n,\left(\hat{\theta}_{k, n}^{b}\right)_{k \in \mathcal{S}}$ is an optimal solution to $B_{n}$;

(b) For all $n,\left(\hat{\theta}_{k, n}^{p}\right)_{k \in \mathcal{S}}$ is an optimal solution to $P_{n}$;

(c) For all $k, p_{k}=\lambda_{k}^{*}+H^{\top}\left(\mu_{k}^{-*}-\mu_{k}^{+*}\right)$, where: (i) $\left(\lambda_{k}^{*}, \mu_{k}^{-*}, \mu_{k}^{+*}\right)_{k \in \mathcal{S}}$ are optimal Lagrange multipliers in ED with surrogate cost functions $c_{n}^{p}\left(q_{k, n}^{p}\right)=$ $\hat{c}\left(q_{k, n}^{p}, \hat{\theta}_{k, n}^{p}\right)$; and (ii) $\lambda_{k}^{*}, \mu_{k}^{-*}, \mu_{k}^{+*}$ are optimal Lagrange multipliers in $F R_{k}$ for all $k \in \mathcal{K} \backslash \mathcal{S}$ with surrogate cost functions $c_{n}^{p}\left(q_{k, n}^{p}\right)=\hat{c}\left(q_{k, n}^{p}, \hat{\theta}_{k, n}^{p}\right)$.

Our main result for this section follows. It highlights that, as a consequence of Theorem 1, the mechanism we propose in this section guarantees that any efficient allocation is supported by a competitive equilibrium.
Proposition 3 (Efficiency). Suppose that the conditions in Theorem 1 hold. Then:

(a) Any competitive equilibrium is efficient.

(b) Any efficient allocation can be sustained by a competitive equilibrium.

Proposition 3 resembles classical welfare theorems, e.g., [32], [34]-[36]. However, it differs from typical competitive equilibria frameworks because peakers are restricted to bidding a single supply function over each economic dispatch interval even though the latter contains multiple fast timescale market instances.

This creates challenges in guaranteeing existence of equilibria and efficiency that do not arise in typical competitive equilibria frameworks. Specifically, the space of bid functions needs to be expressive enough for generators to convey their costs (in multiple fast timescale market instances) using a single bid function. This is not an issue in existing market frameworks where separate bids are collected for separate market instances. Hence our restriction that $s(p)=$ $(1 / \theta) c^{\prime-1}(\theta p)$.

\section{REFERENCES}

[1] A. J. Wood and B. F. Wollenberg, Power Generation, Operation, and Control, 2nd ed. John Wiley \& Sons, Inc., 1996.

[2] A. R. Bergen and V. Vittal, Power Systems Analysis, 2nd ed. Prentice Hall, 2000.

[3] J. Machowski, J. Bialek, and J. Bumby, Power system dynamics: Stability and Control, 2nd ed. John Wiley \& Sons, Inc., 2008.

[4] J. Carpentier, "Optimal power flows," International Journal of Electrical Power \& Energy Systems, vol. 1, no. 1, pp. 3-15, 1979.

[5] D. Kirschen and G. Strbac, Fundamentals of Power System Economics. Wiley Online Library, 2004.

[6] R. Baldick, R. Grant, and E. Kahn, "Theory and application of linear supply function equilibrium in electricity markets," Journal of Regulatory Economics, vol. 25, no. 2, pp. 143-167, 2004.

[7] C. Inc., "Market Optimization Details," http://caiso.com/Documents/ TechnicalBulletin-MarketOptimizationDetails.pdf, November 2009, [Online; accessed Mar-24-2015].

[8] F. C. Schweppe, R. D. Tabors, M. Caraminis, and R. E. Bohn, "Spot pricing of electricity," 1988.

[9] I. Ibraheem, P. Kumar, and D. Kothari, "Recent philosophies of automatic generation control strategies in power systems," Power Systems, IEEE Transactions on, vol. 20, no. 1, pp. 346-357, Feb 2005.

[10] "IEEE Standard Definitions of Terms for Automatic Generation Control on Electric Power Systems," Power Apparatus and Systems, IEEE Transactions on, no. 6, pp. 1356-1364, 1970.

[11] E. Mallada, C. Zhao, and S. H. Low, "Optimal load-side control for frequency regulation in smart grids," in Proc. of Allerton Conference on Communication, Control, and Computing, Monticello, IL, USA, 2014, pp. 731-738

[12] M. Chiang, S. H. Low, A. R. Calderbank, and J. C. Doyle, "Layering as optimization decomposition: A mathematical theory of network architectures," Proceedings of the IEEE, vol. 95, no. 1, pp. 255-312, 2007.

[13] D. P. Palomar and M. Chiang, "Alternative distributed algorithms for network utility maximization: Framework and applications," Automatic Control, IEEE Transactions on, vol. 52, no. 12, pp. 2254-2269, 2007.

[14] _ - "A tutorial on decomposition methods for network utility maximization," Selected Areas in Communications, IEEE Journal on, vol. 24, no. 8, pp. 1439-1451, 2006.

[15] D. W. Cai, C. W. Tan, and S. H. Low, "Optimal max-min fairness rate control in wireless networks: Perron-frobenius characterization and algorithms," in INFOCOM, 2012 Proceedings IEEE. IEEE, 2012, pp. $648-656$.

[16] A. R. Bergen and D. J. Hill, "A structure preserving model for power system stability analysis," IEEE Trans. Power App. Syst., no. 1, pp. 25-35, 1981.

[17] C. Zhao, U. Topcu, N. Li, and S. H. Low, "Design and stability of loadside primary frequency control in power systems," arXiv:1305.0585, 2013.

[18] N. Li, L. Chen, C. Zhao, and S. H. Low, "Connecting automatic generation control and economic dispatch from an optimization view," in Proc. of American Control Conference, Portland, OR, USA, 2014, pp. $735-740$. 
[19] F. Dörfler, J. Simpson-Porco, and F. Bullo, "Breaking the hierarchy: Distributed control \& economic optimality in microgrids," arXiv preprint arXiv:1401.1767, 2014.

[20] C. Zhao, U. Topcu, N. Li, and S. Low, "Design and Stability of Load-Side Primary Frequency Control in Power Systems," Automatic Control, IEEE Transactions on, vol. 59, no. 5, pp. 1177-1189, 2014.

[21] S. You and L. Chen, "Reverse and forward engineering of frequency control in power networks," in Conference on Decision and Control, 2014.

[22] S. Stoft, Power system economics. IEEE Press, 2002.

[23] A. Ehrenmann and K. Neuhoff, "A comparison of electricity market designs in networks," Operations research, vol. 57, no. 2, pp. 274-286, 2009.

[24] R. Baldick, "Electricity market equilibrium models: The effect of parametrization," IEEE Transactions on Power Systems, vol. 17, no. 4, pp. 1170-1176, 2002.

[25] A. D. Papalexopoulos and P. E. Andrianesis, "Performance-based pricing of frequency regulation in electricity markets," Power Systems, IEEE Transactions on, vol. 29, no. 1, pp. 441-449, 2014.

[26] Y. V. Makarov, C. Loutan, J. Ma, and P. de Mello, "Operational impacts of wind generation on california power systems," Power Systems, IEEE Transactions on, vol. 24, no. 2, pp. 1039-1050, 2009.

[27] C. Inc., "Flexible Ramping Products Draft Final Proposal," https://www.caiso.com/Documents/DraftFinalProposal FlexibleRampingProduct_includingFMM-EIM.pdf, December 2014, [Online; accessed Mar-24-2015].

[28] B. Willems, "Modeling cournot competition in an electricity market with transmission constraints," The Energy Journal, pp. 95-125, 2002.

[29] C. Metzler, B. F. Hobbs, and J.-S. Pang, "Nash-cournot equilibria in power markets on a linearized dc network with arbitrage: Formulations and properties," Networks and Spatial Economics, vol. 3, no. 2, pp. 123-150, 2003.

[30] B. F. Hobbs, C. B. Metzler, and J.-S. Pang, "Strategic gaming analysis for electric power systems: An mpec approach," Power Systems, IEEE Transactions on, vol. 15, no. 2, pp. 638-645, 2000.

[31] A. Rudkevich, "On the supply function equilibrium and its applications in electricity markets," Decision Support Systems, vol. 40, no. 3, pp. 409-425, 2005.

[32] R. Johari and J. N. Tsitsiklis, "Parameterized supply function bidding: Equilibrium and efficiency," Operations research, vol. 59, no. 5, pp. 1079-1089, 2011

[33] P. D. Klemperer and M. A. Meyer, "Supply function equilibria in oligopoly under uncertainty," Econometrica: Journal of the Econometric Society, pp. 1243-1277, 1989.

[34] A. Mas-Colell, M. D. Whinston, J. R. Green, et al., Microeconomic theory. Oxford university press New York, 1995, vol. 1.

[35] R. Johari and J. N. Tsitsiklis, "Efficiency of scalar-parameterized mechanisms," Operations Research, vol. 57, no. 4, pp. 823-839, 2009.

[36] G. Wang, M. Negrete-Pincetic, A. Kowli, E. Shafieepoorfard, S. Meyn, and U. V. Shanbhag, "Dynamic competitive equilibria in electricity markets," in Control and optimization methods for electric smart grids. Springer, 2012, pp. 35-62.

\section{APPENDIX}

Proof of Theorem 1. First, by substituting the baseloads' decision constraints (6) in $S Y S T E M$, we can eliminate the variables $q_{k}^{b}$ for all $k \notin \mathcal{S}$. Now, instead of relaxing all the supply-demand balance constraints and line flow constraints, relax only the constraints associated with period $k \in \mathcal{K} \backslash \mathcal{S}$. The remaining constraints (that have not been relaxed) are:

$$
\begin{array}{rr}
\mathbf{1}^{\top}\left(q_{k}^{b}+q_{k}^{p}-d_{k}\right)=0, & k \in \mathcal{S} ; \\
-f \leq H\left(q_{k}^{b}+q_{k}^{p}-d_{k}\right) \leq f, & k \in \mathcal{S} ; \\
0 \leq q_{k}^{p} \leq \bar{q}^{p}, & k \in \mathcal{K} ; \\
0 \leq q_{k}^{b} \leq \bar{q}^{b}, & k \in \mathcal{S} ;
\end{array}
$$

Let $\bar{y}=\left(\lambda_{k}, \mu_{k}^{-}, \mu_{k}^{+}\right)_{k \in \mathcal{K} \backslash \mathcal{S}}$. The Lagrangian associated with relaxing only those afore-mentioned constraints is given by:

$$
\begin{aligned}
\bar{L}(x, \bar{y})= & \sum_{k \in \mathcal{K} \backslash \mathcal{S}} \bar{f}\left(q_{k}^{p},\left(\lambda_{k}, \mu_{k}^{-}, \mu_{k}^{+}\right)\right) \\
& +\sum_{k \in \mathcal{S}} \bar{g}\left(\left(q_{k}^{b}, q_{k}^{p}\right),\left(\lambda_{k^{\prime}}, \mu_{k^{\prime}}^{-}, \mu_{k^{\prime}}^{+}\right)_{k^{\prime} \in \mathcal{K}_{k} \backslash\{k\}}\right),
\end{aligned}
$$

where:

$$
\begin{aligned}
& \bar{f}\left(q_{k}^{p},\left(\lambda_{k}, \mu_{k}^{-}, \mu_{k}^{+}\right)\right) \\
& \quad:=\sum_{n} c_{n}^{p}\left(q_{n, k}^{p}\right)-\lambda_{k} \mathbf{1}^{\top} q_{k}^{p}-\left(\mu_{k}^{-}-\mu_{k}^{+}\right)^{\top} H q_{k}^{p}, \\
& \bar{g}\left(\left(q_{k}^{b}, q_{k}^{p}\right),\left(\lambda_{k^{\prime}}, \mu_{k^{\prime}}^{-}, \mu_{k^{\prime}}^{+}\right)_{k^{\prime} \in \mathcal{K}_{k} \backslash\{k\}}\right) \\
& \quad:=\sum_{n}\left(S c_{n}^{b}\left(q_{n, k}^{b}\right)+c_{n}^{p}\left(q_{n, k}^{p}\right)\right) \\
& \quad-\sum_{k^{\prime} \in \mathcal{K}_{k} \backslash\{k\}} \lambda_{k^{\prime}} \mathbf{1}^{\top} q_{k}^{b}-\sum_{k^{\prime} \in \mathcal{K}_{k} \backslash\{k\}}\left(\mu_{k^{\prime}}^{-}-\mu_{k^{\prime}}^{+}\right)^{\top} H q_{k}^{b} .
\end{aligned}
$$

Hence, SYSTEM is equivalent to the following problem:

$$
\max _{\bar{y}: \mu_{k}^{-} \geq 0, \mu_{k}^{+} \geq 0, k \in \mathcal{K} \backslash \mathcal{S}} \min _{x:(12 \mathrm{a})-(12 \mathrm{~d})} \bar{L}(x, \bar{y}) .
$$

It is clear that the minimization over $x$ separates into subproblems. For each $k \in \mathcal{K} \backslash \mathcal{S}$, we have the following subproblem:

$$
\begin{aligned}
& \bar{F} R_{k}: \quad \text { minimize } \quad \bar{f}\left(q_{k}^{p},\left(\lambda_{k}, \mu_{k}^{-}, \mu_{k}^{+}\right)\right) \\
& \text {over } \quad q_{k}^{p} ; \\
& \text { s.t. } \quad 0 \leq q_{k}^{p} \leq \bar{q}^{p} ; \\
& \text { given } \quad \lambda_{k}, \mu_{k}^{-}, \mu_{k}^{+} .
\end{aligned}
$$

And we have the following subproblem for the remaining variables:

$$
\begin{aligned}
& E D: \operatorname{minimize} \sum_{k \in \mathcal{S}} \bar{g}\left(\left(q_{k}^{b}, q_{k}^{p}\right),\left(\lambda_{k^{\prime}}, \mu_{k^{\prime}}^{-}, \mu_{k^{\prime}}^{+}\right)_{k^{\prime} \in \mathcal{K}_{k} \backslash\{k\}}\right) \\
& \text { over } \quad q_{k}^{p}, q_{k}^{b}, \quad k \in \mathcal{S} ; \\
& \text { s.t. } \quad \mathbf{1}^{\top}\left(q_{k}^{b}+q_{k}^{p}-d_{k}\right)=0, \quad k \in \mathcal{S} ; \\
&-f \leq H\left(q_{k}^{b}+q_{k}^{p}-d_{k}\right) \leq f, \quad k \in \mathcal{S} ; \\
& 0 \leq q_{k}^{p} \leq \bar{q}^{p}, \quad k \in \mathcal{S} ; \\
& 0 \leq q_{k}^{b} \leq \bar{q}^{b}, \quad k \in \mathcal{S} ; \\
& \text { given } \lambda_{k}, \mu_{k}^{-}, \mu_{k}^{+}, \quad k \in \mathcal{K} \backslash \mathcal{S} .
\end{aligned}
$$

Clearly, if $q_{k}^{b}=0$ for all $k \in \mathcal{S}$, then $\overline{E D}$ is equivalent to $E D$ and hence the solution to $E D$ are the optimal quantities $\left(q_{k}^{b *}, q_{k}^{p *}\right)_{k \in \mathcal{S} \text {. Substitute these optimal quantities }}$ into SYSTEM. Note that the remaining optimization over $\left(q_{k}^{p *}\right)_{k \in \mathcal{K} \backslash \mathcal{S}}$ can be separated into independent problems $\left(F R_{k}\right)_{k \in \mathcal{K} \backslash \mathcal{S}}$ where the solution to $F R_{k}$ is the optimal quantity $q_{k}^{p *}$. This proves the sufficiency of condition (a).

Next, we prove the sufficiency of conditions (b) and (c). The constraint (12a) implies that $\bar{g}$ can be rewritten as:

$$
\begin{aligned}
\bar{g} & \left(\left(q_{k}^{b}, q_{k}^{p}\right),\left(\lambda_{k^{\prime}}, \mu_{k^{\prime}}^{-}, \mu_{k^{\prime}}^{+}\right)_{k^{\prime} \in \mathcal{K}_{k} \backslash\{k\}}\right) \\
: & \sum_{n}\left(S c_{n}^{b}\left(q_{n, k}^{b}\right)+c_{n}^{p}\left(q_{n, k}^{p}\right)\right)-\sum_{k^{\prime} \in \mathcal{K}_{k} \backslash\{k\}} \lambda_{k^{\prime}} \mathbf{1}^{\top}\left(d_{k}-q_{k}^{p}\right) \\
& -\sum_{k^{\prime} \in \mathcal{K}_{k} \backslash\{k\}}\left(\mu_{k^{\prime}}^{-}-\mu_{k^{\prime}}^{+}\right)^{\top} H q_{k}^{b} .
\end{aligned}
$$

It is easy to see that $\overline{E D}$ is equivalent to $E D$ under conditions (b) and (c). The separation of the remaining optimization into independent problems $\left(F R_{k}\right)_{k \in \mathcal{K} \backslash \mathcal{S}}$ follows from the same arguments as before. 